\title{
Preferred Sites of Exocytosis and Endocytosis Colocalize during High- But Not Lower-Frequency Stimulation in Mouse Motor Nerve Terminals
}

\author{
Michael A. Gaffield, ${ }^{1}$ Lucia Tabares, ${ }^{2}$ and William J. Betz ${ }^{1}$ \\ ${ }^{1}$ Department of Physiology and Biophysics, University of Colorado Denver, Anschutz Medical Campus, Aurora, Colorado 80045, and ${ }^{2}$ Department of \\ Medical Physiology and Biophysics, School of Medicine, University of Seville, 41009 Seville, Spain
}

The spatial relationship of exocytosis and endocytosis in motor nerve terminals has been explored, with varied results, mostly in fixed preparations and without direct information on the utilization of each exocytic site. We sought to determine these spatial properties in real time using synaptopHluorin ( $\mathrm{spH}$ ) and FM4-64. Earlier we showed that nerve stimulation elicits the appearance of spH fluorescence hot spots, which mark preferred sites of exocytosis. Here we show that nerve stimulation in the presence of the styryl dye FM4-64 evokes hot spots of FM4-64 fluorescence. Their size, density, and rate of appearance are similar to the spH hot spots, but their rate of disappearance after stimulation was much slower $\left(t_{1 / 2} \sim 9 \mathrm{~min}\right.$ vs $\sim 10 \mathrm{~s}$ for $\mathrm{spH}$ hot spots), consistent with FM4-64 spots identifying bulk endocytosis and subsequent slow intracellular dispersion of nascent vesicles. Simultaneous imaging of both fluorophores revealed a strong colocalization of spH and FM4-64 spots, but only during high (100 Hz) stimulation. At $40 \mathrm{~Hz}$ stimulation, exocytic and endocytic spots did not colocalize. Our results are consistent with the hypothesis that hot spots of endocytosis, possibly in the form of bulk uptake, occur at or very near highly active exocytic sites during high-frequency stimulation.

\section{Introduction}

During synaptic transmission at the neuromuscular junction (NMJ), dozens of synaptic vesicles fuse simultaneously with the motor nerve terminal membrane. To maintain a supply of synaptic vesicles, the nerve terminal recovers membrane from the surface via endocytosis [for review, see Wu et al. (2007) and Smith et al. (2008)]. Electron microscopic snapshots of nerve terminals have revealed different modes of endocytosis, including single vesicle retrieval via clathrin-dependent endocytosis (Ceccarelli et al., 1973; Heuser and Reese, 1973; Takei et al., 1996), and uptake of large cisternae or endosomes, which slowly bud synaptic vesicles (Koenig and Ikeda, 1989; Takei et al., 1996; Richards et al., 2000; Teng and Wilkinson, 2000; Holt et al., 2003) [cisternae may maintain a small connection with the plasma membrane (Gad et al., 1998)]. This latter form, often referred to as bulk endocytosis, occurs predominantly during periods of intense activity when a large amount of membrane is added to the surface (Ceccarelli et al., 1972; Heuser and Reese, 1973) [see also $\mathrm{Xu}$ et al. (2002) at the mouse NMJ] and may arise from sites enriched in endocytic proteins (Estes et al., 1996). In CNS

Received Sept. 18, 2009; revised; ; accepted 0ct. 12, 2009.

This work was funded by grants from the National Institutes of Health (5 R01 NS023466) and the Muscular Dystrophy Association (MDA4204). M.A.G. was supported by a National Institutes of Health research training grant (5 T32 NS007083). We thank Steven Fadul for unfailing assistance, Dr. Kristin Schaller for help with genotyping, Dr. Guillermo Alvarez de Toledo and Dr. Rafael Fernandez-Chacón for valuable comments, and Clinton Cave for useful discussions.

Correspondence should be addressed to William J. Betz, Department of Physiology and Biophysics, University of Colorado Denver, RC1 North, P18-7129, P.0. Box6511, MS 8307,Aurora, C0 80045. E-mail: bill.betz@ucdenver.edu. D01:10.1523/JNEUROSCI.4646-09.2009

Copyright $\odot 2009$ Society for Neuroscience ～0270-6474/09/2915308-09\$15.00/0 nerve terminals, bulk endocytosis is activated immediately with intense stimulation (Clayton et al., 2008), but at the snake NMJ, bulk uptake can occur independently of stimulation levels (Teng et al., 2007).

Signs of extensive endocytosis were first noted toward terminal edges, where nerve, muscle, and Schwann cell membranes converge, far away from exocytic sites (Heuser and Reese, 1973). A later report suggested that endocytosis locations may have no spatial preference (Miller and Heuser, 1984). More recently, spatial locations of endocytic sites have been proposed to emerge in close proximity to exocytic sites at the NMJ (Roos and Kelly, 1999; Teng and Wilkinson, 2000).

We sought to address the degree of colocalization of exocytic and endocytic sites in real time in living motor nerve terminals during different stimulation intensity levels. For localizing exocytic sites, we stimulated motor nerve terminals from a mouse expressing synaptopHluorin ( $\mathrm{spH}$ ) (Miesenböck et al., 1998; Ng et al., 2002; Tabares et al., 2007), which produces $\mathrm{spH}$ spots on the terminal surface. We recently showed that $\mathrm{spH}$ moves out of, not into, spots after stimulation, thereby providing strong evidence that the spots are exocytic in origin (Gaffield et al., 2009). Here we report observing similar FM4-64 spots when the dye was present during stimulation. FM dyes, used mostly to label endosomes (Betz et al., 1992, 1996), can also reveal sites of exocytosis (Smith and Betz, 1996) because unlabeled membrane added to the surface by exocytosis adsorbs FM dye from solution (FM dye in solution remains nonfluorescent). However, while $\mathrm{spH}$ spots disappear quickly after stimulation ends (due to internalization and reacidification of endosomes, which quenches the $\mathrm{spH}$ fluorescence), FM4-64 fluorescence, which is not acid-quenched, re- 

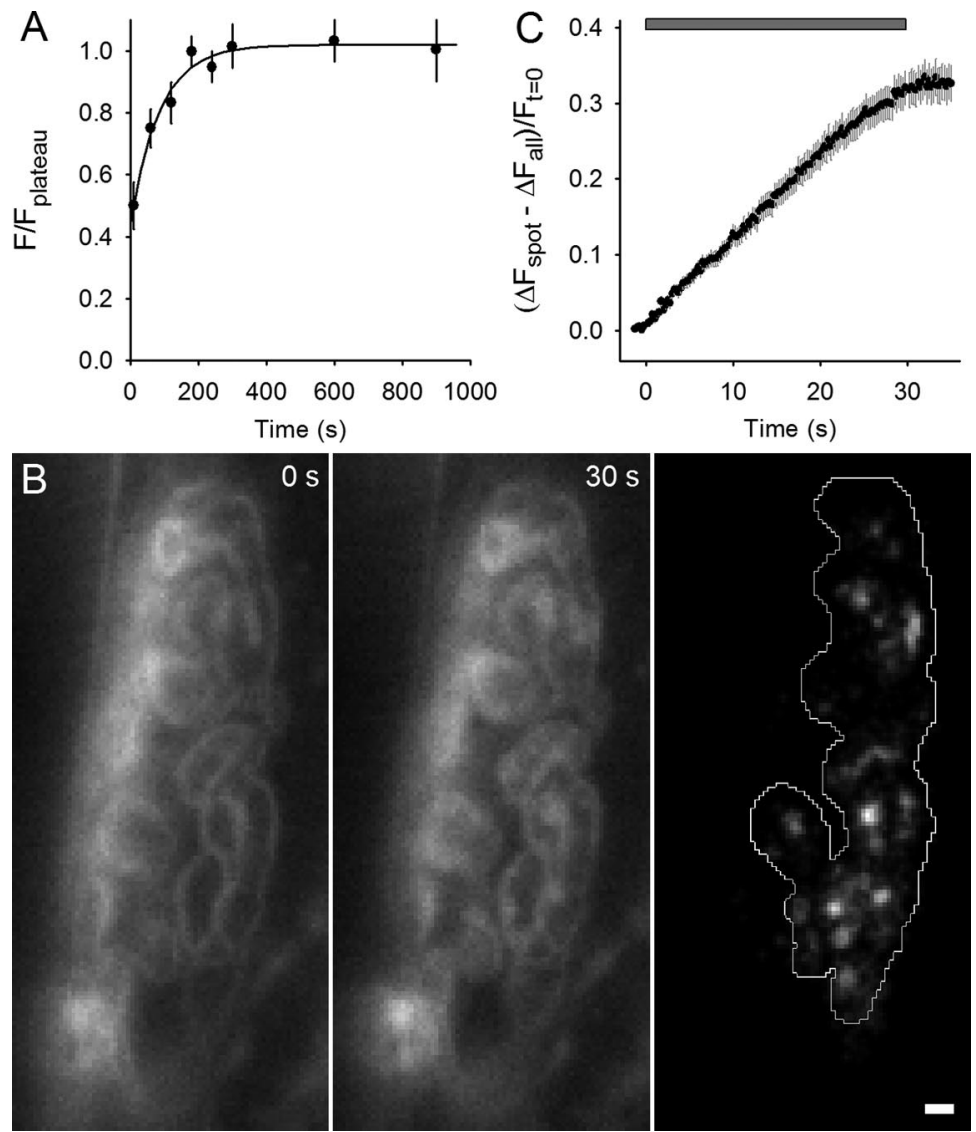

Figure 1. FM4-64 spots appear during $100 \mathrm{~Hz}$ stimulation. A, Average FM4-64 fluorescence measured versus time in resting terminals. FM4-64 $(3 \mu \mathrm{M})$ was added at $t=0$. Filled circles indicate measurements and the solid line a single-exponential best fit to the data (time constant $=87 \mathrm{~s}$ ). Experiments for FM4-64 spot data were started $\sim 120 \mathrm{~s}$ after dye application. $\boldsymbol{B}$, Left, NMJ in the presence of FM4-64, which shows outline of nerve terminal before stimulation. Middle, Same NMJ after $30 \mathrm{~s}, 100 \mathrm{~Hz}$ nerve stimulation; fluorescent spots have appeared. Right, Difference between middle and left panels revealing FM4-64 spots; the terminal is outlined. Scale bar, $2 \mu \mathrm{m}$. C, The change in fluorescence intensity for spots compared with the whole terminal as a fraction of initial fluorescence is plotted versus time. During the time indicated by the gray bar, $100 \mathrm{~Hz}$ stimulation was delivered.

mained intact long after stimulation ceased. Thus, the FM4-64 spots, which dissipated only as the nascent endosomes and vesicles dispersed in the cytoplasm, mark endocytosis hot spots. We imaged both fluorophores simultaneously and report that FM4-64 spots arose close to $\mathrm{spH}$ spots during intense but not moderate stimulation.

\section{Materials and Methods}

Acute muscle preparation. spH mice (Tabares et al., 2007) or wild-type mice $(\mathrm{C} 57 \mathrm{BL} / 6)$ were housed, handled, and bred in accordance with the University of Colorado Denver Institutional Animal Care and Use Committee. The levator auris longus muscle was dissected from adult mice as described previously (Angaut-Petit et al., 1987) and pinned in a Sylgardlined chamber. For nerve stimulation, the nerve was drawn into a suction electrode. The perfusing solution contained (in $\mathrm{mM}$ ) $137 \mathrm{NaCl}, 5 \mathrm{KCl}, 1.8$ $\mathrm{CaCl}_{2}, 1 \mathrm{MgSO}_{4}, 12 \mathrm{NaHCO}_{3}, 1 \mathrm{NaH}_{2} \mathrm{PO}_{4}$, and 11 glucose with $\mathrm{pH} 7.4$ and bubbled with $95 \% \mathrm{O}_{2} / 5 \% \mathrm{CO}_{2}$. During experiments, gravity fed perfusion was maintained at a rate of $\sim 1 \mathrm{ml} / \mathrm{min}$ into a volume of $\sim 5 \mathrm{ml}$. Temperature was maintained at $36 \pm 1^{\circ} \mathrm{C}$ using a dish heater DH-35 and inline heater SF-28 controlled and monitored by a dual automatic temperature controller TC-344B, all from Warner Instruments. Muscle contraction was blocked by adding $3 \mu \mathrm{M}$ curare (Sigma). FM4-64 was added directly to the chamber with an initial chamber concentration of $3 \mu \mathrm{M}$ for characterization experiments. For experiments presented in Figure 3, we used a $1 \mu \mathrm{M}$ concentration after changing filter sets to optimize the FM4-64 signal (see below). The FM4-64 concentration was reduced again to $0.75 \mu \mathrm{M}$ for Figures $4-7$. This reduction did not significantly change the FM4-64 spot characteristics after $100 \mathrm{~Hz}$ stimulation (data not shown). To measure synaptic vesicle cluster sizes, terminals were loaded at room temperature with $3.2 \mu \mathrm{M}$ FM4-64 or FM1-43 and stimulated for 1800 shocks at either 10, 30, or $100 \mathrm{~Hz}$. While these stimulation protocols may result in up to a $25 \%$ difference in terminal intensity levels (Gaffield and Betz, 2007), all resulted in significantly larger cluster sizes when compared with FM4-64 spots (data not shown). For simplicity, the data for all loading frequencies were pooled for plotting and analysis (Fig. 2B). Electrophysiological recordings (Fig. $3 B$ ) were performed as described previously (Gaffield and Betz, 2007). Briefly, we collected miniature end plate potentials and end plate potentials in $\mu$-conotoxin, then calculated quantum content according to Martin (1955)

Imaging and stimulation. Imaging was performed on a Leica DM 4000B microscope with a Leica EL 6000 light source. For the spH signal, we used a Leica L5 filter cube [bandpass (BP) 480/40 excitation, 505 dichroic, and BP 527/30 emission]. For the FM4-64 signal, we used either a Leica N3 filter cube (BP 546/12 excitation, 565 dichroic, and BP 600/40 emission) (Figs. 1,2) or a Leica TX2 filter cube (BP 560/40 excitation, 595 dichroic, and BP 645/75 emission) (Figs. 3-7). For two-color experiments, the two different channels were collected sequentially with the filter set change taking $\sim 500 \mathrm{~ms}$. Images were focused using a $63 \times$, 0.9 numerical aperture water-immersion objective from Zeiss. Images were collected using an Andor iXon EM+ DU-897E camera cooled to $-55^{\circ} \mathrm{C}$ and controlled using Andor's Solis Software, version 4.6.5. Pixels were $2 \times 2$ binned resulting in $270 \mathrm{~nm}$ pixels. For nerve stimulation, trains were generated using an A310 Accupulser and delivered using an A365 Stimulus Isolator, both from World Precision Instruments. Precise timing control between imaging and stimulation was directed from software custom written in Matlab (MathWorks) and sent to the instrumentation via a DAQ Card 6036E and a BNC-2090 board, both from National Instruments.

Image analysis and modeling. All analysis was performed using customwritten Matlab code. Terminals were first masked by hand drawing the terminal outline. Next we subtracted background fluorescence from nonterminal areas. For automated spot detection, we created an image with pixel values indicating the fluorescence change during stimulation $\left(F_{30 \mathrm{~s}}-F_{0 \mathrm{~s}}\right)$. Then we identified all local peaks (pixels brighter than all eight surrounding pixels). The peaks were sorted by intensity, then, starting with the brightest peak, we identified all connected pixels with at least $75 \%$ of the peak's intensity. The resulting pixel grouping became the first spot. This process continued for all of the peaks. If any pixels in a grouping overlapped with another spot (having a brighter peak intensity), then the spot was excluded. Finally, any spots that comprised fewer than three pixels were excluded from further analysis. To find the distance to the nearest terminal edge, we drew an outline of the terminal's edges by tracing the FM4-64 fluorescence observable before stimulation. For nearest-neighbor detection, the center of mass for each identified spot was calculated and then compared with all other centers of mass (for both spots of similar fluorescence and different fluorescence). For randomly generated spot data, spots were randomly placed within terminals whose shapes were taken from actual data and using the spot density calculated for each terminal. Random spots always had a center of mass centered on 
a pixel. One thousand spot trials were averaged for each terminal shape to get the average random distribution. The time course of FM4-64 spot dispersion was estimated using linear fits to the recovery phase of both spot and nonspot traces (Fig. 3A). To estimate the amount of exocytosed membrane visible with $\mathrm{spH}$ at the end of stimulation, we used end plate potentials and quantal measurements (Gaffield and Betz, 2007) to calculate the number of vesicles fused with each shock. The total release was simply a sum of the total quanta. After fusion, each vesicle was endocytosed and reacidified with a time constant based on spH recovery.

Statistical analysis was performed using Excel (Microsoft), plots and curve fitting were done using SigmaPlot (Systat Software). All experimental conditions were performed on at least three muscles; trial numbers are listed in the text or figure legends. All error bars indicate SEM. Statistical difference was tested using the Student's $t$ test with $p$ values $<0.05$ indicating significance.

\section{Results \\ Imaging of FM4-64 uptake during $100 \mathrm{~Hz}$ stimulation reveals \\ fluorescent spots}

We used a pipette to add and mix FM4-64 with the normal saline solution in the experimental chamber to a final concentration of $\sim 3 \mu \mathrm{M}$. FM dye fluorescence increases greatly as it partitions into surface membranes and as shown in Figure $1 A$, the fluorescence of terminals rose with a time constant of $87 \mathrm{~s}$. The preparation was slowly perfused with a solution that did not contain FM dye, so the FM4-64 concentration in the dish became dilute over time. Nerve terminal fluorescence, however, remained steady for at least 10 min (Fig. 1A), during which time experimental measurements were made. Figure $1 B$ (left) shows a typical terminal $\sim 2 \mathrm{~min}$ after the dye was added. The terminal outline is clearly visible. We then delivered a train of stimuli ( $100 \mathrm{~Hz}$ for $30 \mathrm{~s}$ ) to the nerve. The image taken immediately after the train (Fig. $1 B$, middle) shows the emergence of distinct fluorescent spots. The difference image Figure $1 B$ (right) shows the spots more clearly. The mean time course of appearance of 84 FM4-64 spots from nine terminals, shown in Figure $1 C$, revealed a rapid onset with fluorescence accumulating at $\sim 1 \%$ of initial intensity levels per second above the terminal average. The rise in fluorescence ended with the end of stimulation.

\section{FM4-64 spots and spH spots have similar characteristics}

In experiments involving imaging with one but not both fluorophores, we noted that FM4-64 spots were similar to spH spots in many aspects (Fig. $2 \mathrm{~A}$, different preparations). To quantify characteristics, we identified spots using an automatic spot-finding routine (see Materials and Methods). Examples are shown in Figure $2 A$ (bottom). Using this process, we measured several
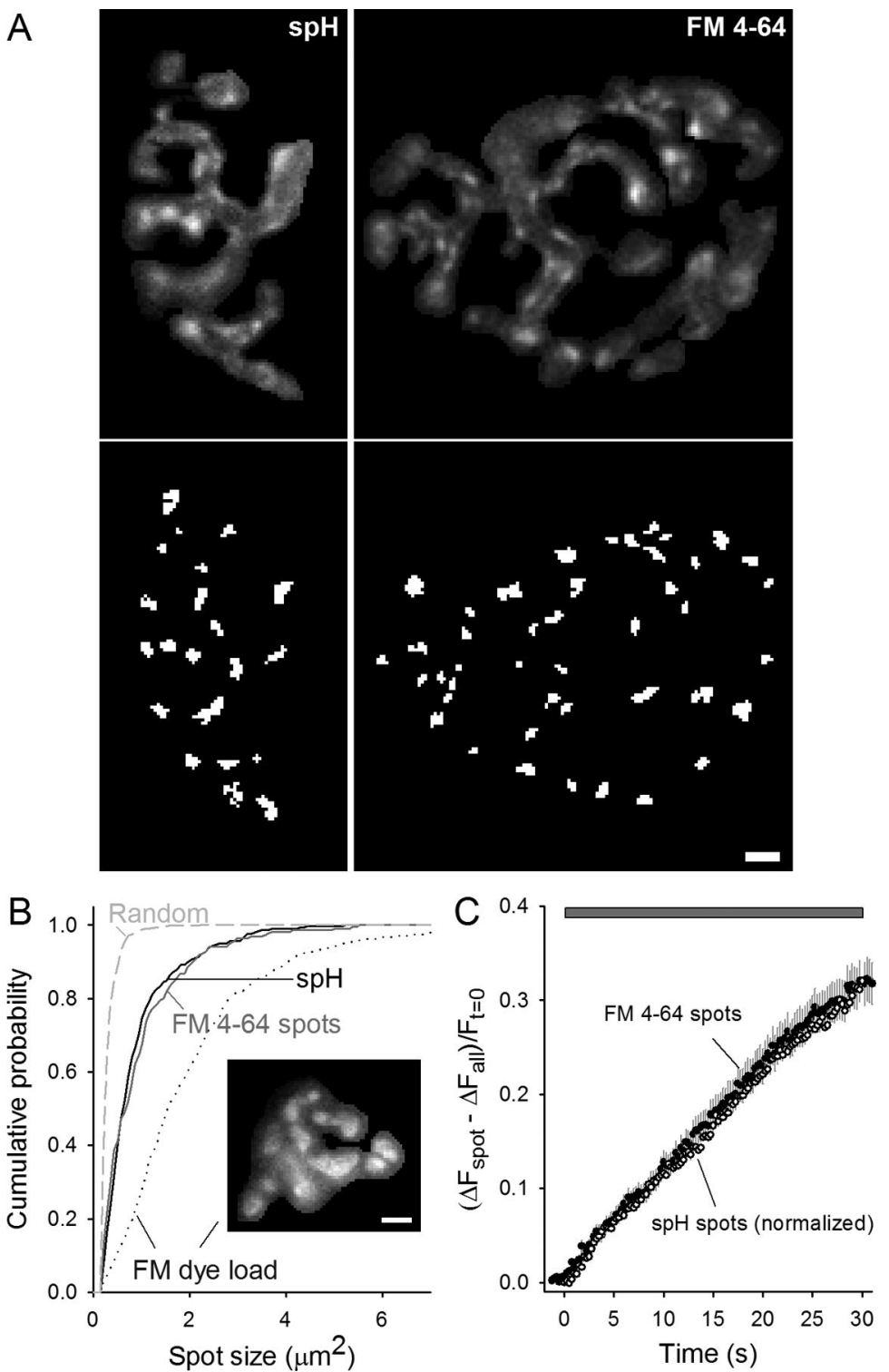

Figure 2. FM4-64 spots and spH spots have similar properties. $\boldsymbol{A}$, Top, Typical difference in spH or FM4-64 fluorescence intensity after a $30 \mathrm{~s}, 100 \mathrm{~Hz}$ stimulation (different preparations). Bottom, Spots identified in the top images by the automatic spot-finding M4-64 spot sizes (solid gray line). For comparison to the overall synaptic vesicle population, the synaptic vesicle distribution was end mixing terminal pixel locations. Data are averaged from 298 spH spots, 220 FM4-64 spots, and 466

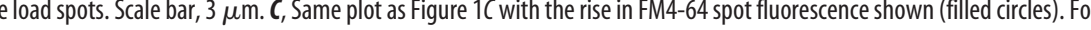
comparison, peak spH spot fluorescence was normalized to match the peak FM4-64 spot fluorescence and plotted (open circles). The mean time to half maximum was $14.5 \pm 1.3 \mathrm{~s}$ and $13.3 \pm 0.6 \mathrm{~s}$ for spH and FM4-64 spots, respectively $(p=0.38$, Student's $t$ test). The gray bar indicates $100 \mathrm{~Hz}$ stimulation.

different spot characteristics. Spot densities were not significantly different $\left(8.9 \pm 0.6\right.$ per $100 \mu \mathrm{m}^{2}$ for $\mathrm{spH}$ and $8.3 \pm 1.1$ per 100 $\mu \mathrm{m}^{2}$ for FM4-64). The distances from spots to nerve terminal edges also were not significantly different $(0.75 \pm 0.10 \mu \mathrm{m}$ for $\mathrm{spH}$ and $0.77 \pm 0.04 \mu \mathrm{m}$ for $\mathrm{spH})$, nor were they significantly different from randomly placed spots $(0.75 \pm 0.04 \mu \mathrm{m})$. Figure $2 \mathrm{~B}$ (solid lines) shows that FM4-64 and $\mathrm{spH}$ spot sizes were not significantly different. For comparison, the sizes of synaptic vesicle clusters in terminals fully loaded with FM dye are much larger than the spH and FM4-64 spots (Fig. 2 B, inset and dotted line). Note that the FM4-64 distribution shown in the solid gray line 

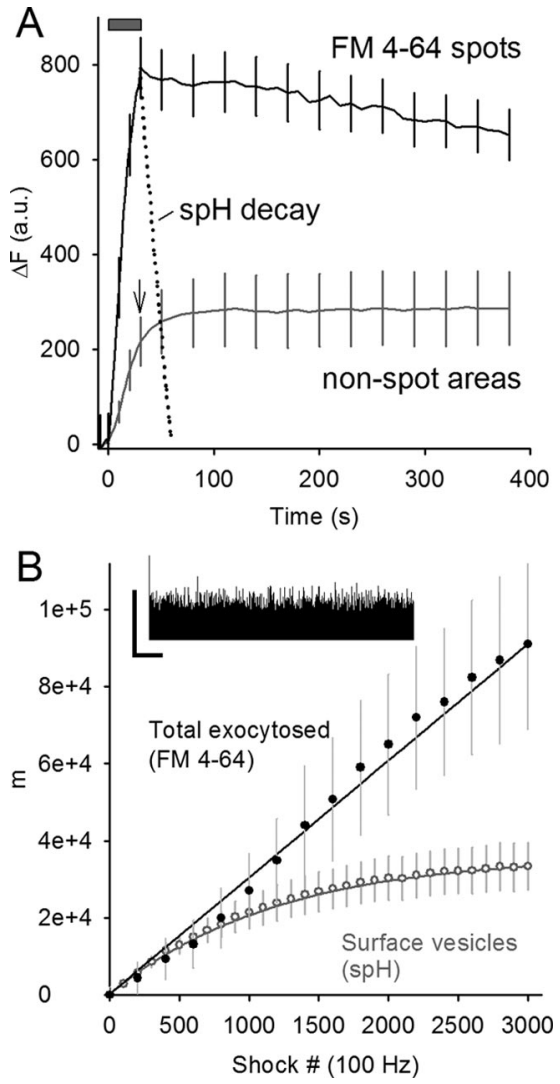

Figure 3. FM4-64 spots persist well after spH endocytosis and reacidification. $\boldsymbol{A}$, Raw change in fluorescence (in arbitrary units) is plotted versus time for either FM4-64 spots (black line) or nonspot areas of the terminal (gray line; arrow marks end of stimulus train). Fluorescence in the FM4-64 spots dispersed slowly after stimulation (train duration indicated by gray bar), particularly when compared with the time course of synaptic vesicle endocytosis and reacidification (spH fluorescence, dotted line). Fluorescence in FM4-64 spots was estimated to match nonspot area fluorescence $\sim 17$ min after stimulation. Data are averaged from five different terminals. For clarity, error bars are shown for every fifth data point. $\boldsymbol{B}, \mathrm{spH}$ labels $35 \%$ of the vesicles labeled by FM4-64 at the end of $100 \mathrm{~Hz}$ stimulation. Inset, Electrophysiological recording of EPPs during $100 \mathrm{~Hz}$ stimulation. The vertical calibration bar indicates the peak amplitude of the first EPP, and the horizontal calibration bar indicates $2 \mathrm{~s}$. Main plot, Quanta $(\mathrm{m})$ are plotted versus shock number. The upper solid line indicates the summed quantal release from EPPs. The lower line indicates the "surface vesicles" or those expected to be visible with spH fluorescence based on a simple model using the spH recovery time constant (see Materials and Methods). Actual FM4-64 fluorescence was scaled to match EPP data (filled circles). The gray circles show the average $\mathrm{spH}$ fluorescence rise during stimulation after scaling to the theoretical surface vesicle number (lower solid line).

would eventually turn into the dotted line after a 15-20 min wash. Finally, the dashed line in Figure $2 B$ shows the size distribution of "spots" in difference images in which we randomly mixed pixels before running the automatic spot-finding routine. Their much smaller sizes show that the spots that we observed were real, and not simply extracted out of the noise. Figure $2 C$ shows that the time courses of emergence of spH and FM4-64 spots were nearly identical on the time scale of seconds. In summary, FM4-64 and $\mathrm{spH}$ spot densities, distances from edge, sizes, and time courses were not significantly different.

\section{FM4-64 spots disperse slowly after stimulation}

Figure $3 A$ shows that FM4-64 spots (black solid line) decay much more slowly than $\mathrm{spH}$ fluorescence $($ dotted line; time constant $=$ $12 \mathrm{~s}$ ). The $\mathrm{spH}$ decay reflects internalization of $\mathrm{spH}$, reacidification of synaptic vesicles or endosomes, and quenching of $\mathrm{spH}$. We analyzed 58 total FM4-64 spots identified in five different terminals and found only one spot with a decay half-time consistent with $\mathrm{spH}$ recovery ( $<30 \mathrm{~s})$. On average, the FM4-64 spots required $\sim 15-20 \mathrm{~min}$ to disperse completely after stimulation ended. As discussed later, the FM4-64 spot decay might reflect the slow budding of vesicles from cisternae, which then move away from the spot into the larger vesicle pool.

The solid gray line in Figure 3A shows the FM4-64 fluorescence change in nonspot regions. The arrow marks the end of the stimulation period. Fluorescence in nonspot areas (which comprised $>90 \%$ of the total terminal area) increased slightly after stimulation ended, reaching levels approximating the overall FM4-64 loaded into the terminal. At the end of the stimulus train, the fluorescence in spots accounted for $20.2 \pm 3.1 \%$ of the total fluorescence contained within the terminal even though the spots comprised only $6.7 \pm 1.5 \%$ of the terminal area, indicating a threefold enrichment of membrane in FM4-64 spot locations.

\section{The majority of vesicles exocytosed during stimulation are internalized by the end of stimulation}

At this point we wondered whether the spH and FM4-64 signals should track together. We know that FM dye will partition into any membrane exposed to FM dye in the extracellular solution, thus labeling all exocytosed membrane independently of endocytosis (cf. Smith and Betz, 1996). On the other hand spH fluoresces only when on the surface or in recently endocytosed structures; therefore, the two signals might provide complementary rather than duplicate information. We tested this prediction by calculating the expected difference between the two signals at the end of stimulation-specifically how much exocytosed membrane would be visible with FM4-64 but dark in the spH channel (Fig. 3B).

We first measured the amount of total exocytosis using electrophysiological recordings of end plate potentials (EPPs). The summed quantum contents were then compared with the rate of FM4-64 fluorescence change for the whole terminal. We saw no difference in the exocytosis rate (upper solid line and filled circles, respectively, in Fig. 3B), suggesting that all exocytosed vesicles were labeled by FM4-64. Next, assuming that the time constant of endocytosis/reacidification during stimulation was the same as after stimulation ended $(\sim 12 \mathrm{~s})$, we estimated that only $\sim 35 \%$ of all exocytosed vesicles would be visible in the $\mathrm{spH}$ channel at the end of stimulation (Fig. 3B, lower solid line). The actual $\mathrm{spH}$ fluorescence change during $100 \mathrm{~Hz}$ stimulation (open circles) could be fitted well with this model using a single scaling factor. Comparing the FM4-64 and spH data points at the end of stimulation, the nearly threefold higher FM4-64 signal means that approximately two-thirds of the FM4-64 signal arose from internalized membrane.

\section{spH and FM4-64 spots evoked by intense}

\section{stimulation colocalize}

For the previous experiments, we had imaged spH and FM4-64 spots separately. Naturally, it was of interest to determine whether the spH spots and the FM4-64 spots colocalized, particularly since the signals were similar in so many aspects (Fig. 2), but provided different information on surface and internalized membrane (Fig. 3). We therefore imaged both fluorescence signals simultaneously in the same terminal. To minimize potential interactions between spH and FM4-64, we reduced the FM4-64 concentration to $0.75 \mu \mathrm{M}$. At the end of the stimulus train both spH and FM4-64 spots were visible; four examples are shown in Figure 4. In overlaid images (third column) spH spots (green) 
seemed to be in close proximity to a FM4-64 spots (red). For example, point \#1 marks an $\mathrm{spH}$ spot flanked by two FM4-64 spots. However, there were exceptions. For example, some spH spots appeared without a nearby FM4-64 spot (Fig. 4, point \#2), and vice versa (point \#3, see Discussion).

We quantified the colocalization in $\mathrm{spH}$ and FM4-64 signals in two ways. First, we identified spot locations (Fig. 4, fourth column) and measured the center of mass locations for each $\mathrm{spH}$ and FM4-64 spot. We then measured the nearest-neighbor distances for FM4-64 spots to other FM4-64 spots (Fig. 5A, open circles). The nearest-neighbor distribution had a lower limit of $\sim 1 \mu \mathrm{m}$ due to the properties of the automatic spot detection routine (smaller separations were grouped into single spots; see Materials and Methods). For comparison, we randomly placed spots in the terminals (see Materials and Methods) and the average distribution (Fig. $5 A$, solid gray line) differed little from the observed (dashed lines show 95\% interval). Similar results were obtained with $\mathrm{spH}$ spots (data not shown). These results suggest that both spH and FM4-64 spots were distributed randomly throughout the terminal. We then measured the nearest-neighbor distribution for FM4-64 spots compared with spH spots (Fig. $5 B$ ). In these cases, spot centers could fall within the same pixel because two different spot types were analyzed. The resulting nearest-neighbor distances were considerably smaller than expected by random spot placement (Fig. $5 B$, compare open circles and solid gray line). Approximately $30 \%$ of FM4-64 spots had a corresponding $\mathrm{spH}$ spot centered within one pixel's distance $(270 \mathrm{~nm}$; indicated by vertical gray lines in Fig. $5 A, B)$, and $70 \%$ of FM4- 64 spots had an spH spot within $1 \mu \mathrm{m}$; only $27 \%$ of FM4-64 spots would be expected to have an spH spot so close by chance alone.

Our second method for comparing FM4-64 and spH spatial overlap did not rely on spot detection. Instead we sorted each pixel within the terminal (for each fluorescence channel) according to its fluorescence change during the train $\left(F_{30 \mathrm{~s}}-F_{0 \mathrm{~s}}\right)$. We then calculated the fractional overlap in pixel locations for the two channels as we varied the percentage of total pixels analyzed. The results are plotted in Figure $5 C$ (black line). We found substantially more overlap in FM4-64 and spH fluorescence than predicted by chance (dashed line). For example, the $10 \%$ of pixels that brightened the most in each channel (which are a good approximation of the spots themselves) (Gaffield et al., 2009) overlapped $46.5 \pm 3.0 \%$, much more than expected by chance $(10 \%)$. As a test for the quality of the alignment, we shifted the FM4-64 channel by one pixel ( $x$ and $y$ ) and repeated the calculation. The result (Fig. 5C, solid gray line) showed a small degradation of the colocalization, suggesting that the original alignment was optimal. In summary, during $100 \mathrm{~Hz}$ stimulation, spH spots and FM4-64 spots arise at sites scattered randomly throughout the terminal, but in close proximity to one another.

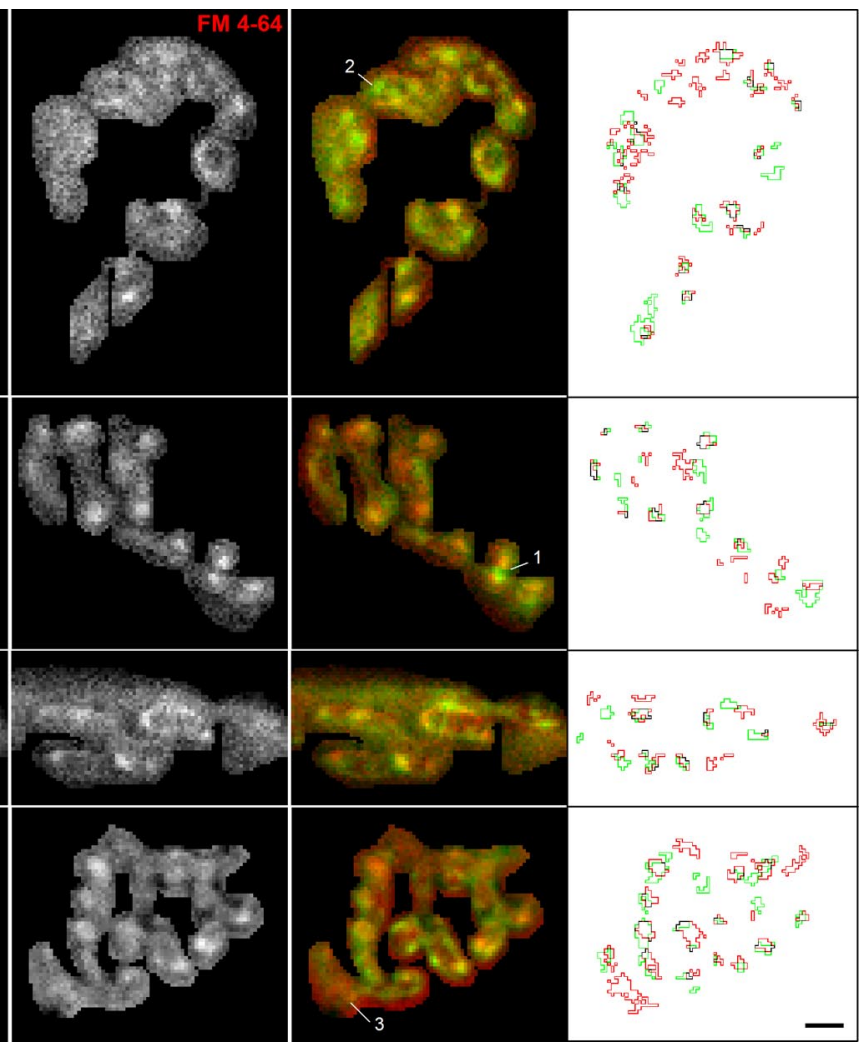

Images show close proximity of spH and FM4-64 spots. Images showing the fluorescence change in response to a $30 \mathrm{~s}$ labeled 1), an spH spot without a nearby FM4-64 spot (spot labeled 2), and an FM4-64 spot without a nearby spH spot (spot labeled 3). The fourth column shows the spot outlines for spH (green) and FM4-64 (red). Scale bar, $3 \mu \mathrm{m}$.

\section{spH and FM4-64 spots are less well correlated at} moderate frequencies

We next asked whether spots would colocalize during less intense stimulation $(40 \mathrm{~Hz}$ instead of $100 \mathrm{~Hz})$. We delivered a $30 \mathrm{~s}, 40 \mathrm{~Hz}$ stimulation train, and collected both $\mathrm{spH}$ and FM4-64 fluorescence as before. Two examples are shown in Figure 6. We were able to identify and characterize $\mathrm{spH}$ spots after this stimulation (Fig. 6, first column), although they were not as prominent as at $100 \mathrm{~Hz}$. The change in fluorescence in the spots was $1.50 \pm 0.04$ times the terminal average after $40 \mathrm{~Hz}$ stimulation compared with $1.87 \pm 0.04$ times the terminal average after $100 \mathrm{~Hz}$ stimulation ( $p<0.05$, Student's $t$ test). While reducing the stimulation frequency did reduce the $\mathrm{spH}$ spot intensity, the $\mathrm{spH}$ spot properties were similar to those at higher frequency. The average spot size was $0.80 \pm 0.06 \mu \mathrm{m}^{2}$ at $40 \mathrm{~Hz}$ (compared with $0.92 \pm 0.05$ $\mu \mathrm{m}^{2}$ for $100 \mathrm{~Hz} ; p=0.13$, Student's $t$ test), and the spot density was $11.33 \pm 1.82$ per $100 \mu \mathrm{m}^{2}$ of terminal area (compared with $8.91 \pm 0.64$ per $100 \mu \mathrm{m}^{2}$ for $100 \mathrm{~Hz} ; p=0.28$, Student's $t$ test). The time constant for $\mathrm{spH}$ recovery was $9.6 \mathrm{~s}$ after $40 \mathrm{~Hz}$ stimulation, modestly faster than after $100 \mathrm{~Hz}$.

In contrast to $\mathrm{spH}$ spots, FM4-64 spots were much less evident after $40 \mathrm{~Hz}$ stimulation (Fig. 6, second column). The average fluorescence increase in FM4-64 spots was only $5 \pm 0.5 \%$ above the initial fluorescence (compared with $32 \pm 2.2 \%$ for $30 \mathrm{~s}, 100$ $\mathrm{Hz}$ and $14 \pm 1.3 \%$ for $12 \mathrm{~s}, 100 \mathrm{~Hz} ; p<0.05$, Student's $t$ test). The average FM4-64 spot size was $3.10 \pm 0.51 \mu \mathrm{m}^{2}$, over three times the spot size at $100 \mathrm{~Hz}\left(0.98 \pm 0.06 \mu \mathrm{m}^{2} ; p<0.05\right.$, Student's $t$ test), indicating a much more diffuse FM4-64 signal. Consequently, the FM4-64 spot density decreased, although not sig- 

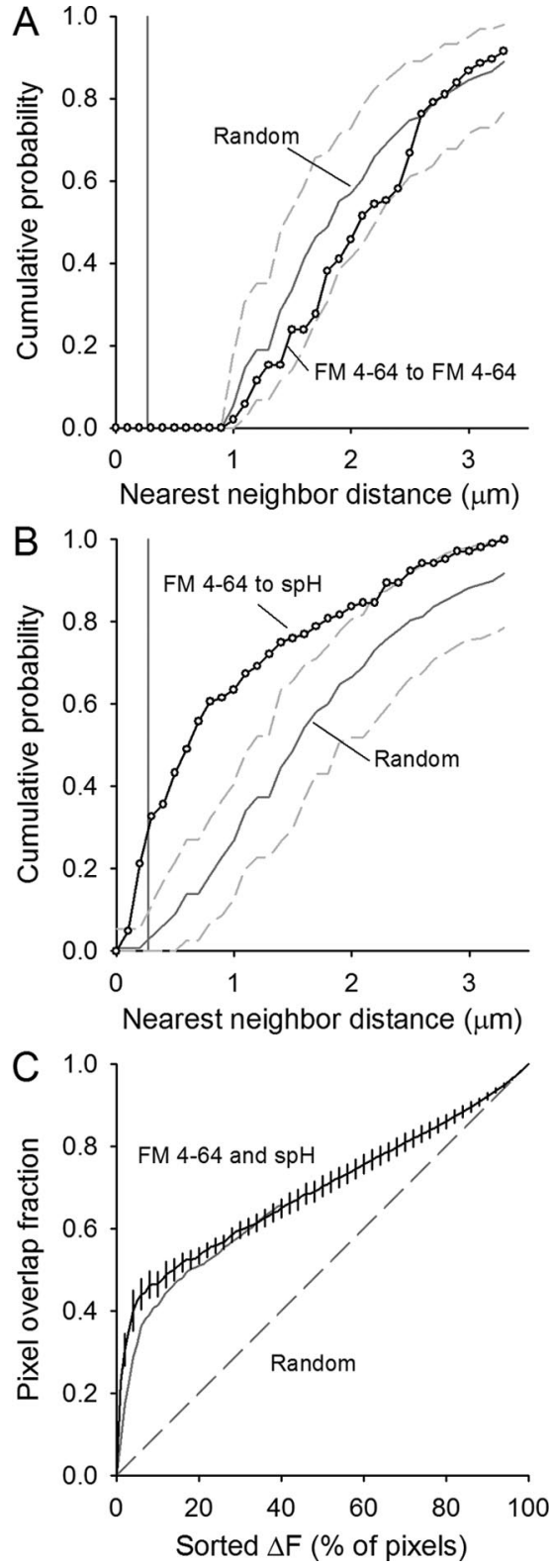

Figure 5. Quantification of spH and FM4-64 spot overlap. A, Cumulative probability plot of the nearest-neighbor distances for FM4-64 spots visible after a $30 \mathrm{~s}, 100 \mathrm{~Hz}$ stimulation (open circles). The automatic spot detection prevents any two similar spot types from existing within $\sim 1 \mu \mathrm{m}$ of each other (pixel size $=270 \mathrm{~nm}$, vertical gray line). Spots placed randomly in the terminal (1000 iterations) have an average distribution indicated by the solid gray line with $95 \%$ of all random trials falling between the dashed gray lines. Data represent 105 spots from five different terminals. $\boldsymbol{B}$, Cumulative probability plot of the nearest-neighbor distances for FM4-64 spots to spH spots (open circles). Spots centered within one pixel distance lie to the left of the vertical gray line $(270 \mathrm{~nm})$. Spots placed randomly in the terminal 1000 times have an average distribution indicated by the dark gray line with $95 \%$ of all random trials falling within the dashed gray lines. C, For each fluorescent label, the total fluorescence change in each pixel was determined, then pixels were sorted based on their relative fluorescence changes during the $30 \mathrm{~s}, 100 \mathrm{~Hz}$ train. The $x$-axis shows the percentage of those sorted pixels (for example, $10 \%$ represents the $10 \%$ of all terminal pixels with the largest fluorescence change during stimulation). The overlap in pixel locations for spH and FM4-64 fluorescence is plotted from 0\% to 100\% (black line with error bars). Overlap is considerably more than by random chance (dashed line). Some of the overlap is lost if the FM4-64 channel is shifted by one pixel in both the $x$ and $y$ directions (solid gray line), suggesting that the original alignment between the two channels was optimal. nificantly, to $6.07 \pm 1.71$ per $100 \mu \mathrm{m}^{2}$ of terminal area (compared with $8.35 \pm 1.12$ per $100 \mu \mathrm{m}^{2}$ for $100 \mathrm{~Hz} ; p=0.28$, Student's $t$ test).

We tested for colocalization of $\mathrm{spH}$ and FM4-64 signals as before. The third column in Figure 6 shows that the spH and FM4-64 signals did not overlap much as indicated by the predominantly green and red color of the overlays. Spot locations also showed considerably less overlap at $40 \mathrm{~Hz}$ (Fig. 4, fourth column). In fact, when we quantified the colocalization (Fig. 7), the results were no different from independent and random spacing. We observed a random-like distribution for $\mathrm{spH}$ spots alone (Fig. 7A), as with $100 \mathrm{~Hz}$ stimulation. In contrast to observations at $100 \mathrm{~Hz}$, however, the spH spots at $40 \mathrm{~Hz}$ had an FM4-64 spot nearest-neighbor distribution similar to randomly placing two different spot groups within the terminal (Fig. $7 B$, compare with Fig. $5 B$ ). Finally, the spH/FM4-64 overlap in sorted pixel intensities was much closer to randomness at $40 \mathrm{~Hz}$ than at $100 \mathrm{~Hz}$ (Fig. 7C, compare with Fig. 5C).

Immediately upon exocytosis of vesicles lacking FM dye, the two fluorescence signals should colocalize as $\mathrm{spH}$ fluorescence increases and FM dye partitions into the unlabeled membrane. Thus, we might have expected more colocalization after $40 \mathrm{~Hz}$ stimulation. We looked for this extra fluorescence by comparing the change in FM4-64 fluorescence in spH spots to the change in fluorescence of all nonspot areas (neither in $\mathrm{spH}$ spots nor in FM4-64 spots). Indeed, we found a 1.25-fold increase. However, even though we could observe the extra signal, we did not identify these fluorescence increases as spots. The reason was that the FM4-64 fluorescence in FM4-64 spots rose 2.76-fold over the rest of the terminal, far above the $\mathrm{spH}$ spot locations $(p<$ 0.05; Student's $t$ test). In other words, the sites of endocytosis at $40 \mathrm{~Hz}$ were different from the sites of exocytosis. Thus, the colocalization of spH and FM4-64 hot spots depends on the stimulation frequency, with stronger correlation occurring at higher frequencies.

\section{Discussion}

We used two fluorophores (spH and FM4-64) to examine simultaneously the sites of exocytosis and endocytosis in mouse motor nerve terminals. We quantified characteristics of each spot type and found that spH and FM4-64 spots colocalized at high, but not moderate stimulation frequencies. The $\mathrm{spH}$ fluorescence indicates surface vesicle levels because vesicle reacidification is much faster (time constant of 1-2 s) (Balaji et al., 2008; Zhang et al., 2009) than our measured recovery time constant of 12 s. Only vesicles yet to complete endocytosis should fluoresce. We have previously provided evidence for $\mathrm{spH}$ spots as sites of increased exocytosis (Gaffield et al., 2009). In brief, the $\mathrm{spH}$ in $\mathrm{spH}$ spots diffused out of the spots into surrounding areas before endocytosis. FM4-64 [a lipophilic dye that fluoresces strongly in lipids (Betz and Bewick, 1992; Betz et al., 1992)], on the other hand, provides a measure of the cumulative amount of exocytosis without regard for the endocytosis state of each vesicle (Smith and Betz, 1996). Because the equivalent of nearly two-thirds of all the exocytosed vesicle membrane was internalized at the end of stimulation (Fig. 3B) [vesicle identity may be lost on the surface (Fernández-Alfonso et al., 2006; Wienisch and Klingauf, 2006; Tabares et al., 2007)], the change in FM4-64 at the end of stimulation preferentially identified endocytosed structures. Ideally, we should be able to match the FM4-64 and spH signals by applying an ATPase inhibitor to prevent vesicle reacidification (Sankaranarayanan and Ryan, 2001), and thus prevent the loss of spH fluorescence. Unfortunately, under our experimental condi- 
tions $\left(37^{\circ} \mathrm{C}\right)$, we observed a rapid rise in resting vesicle fluorescence shortly after applying the ATPase inhibitor folimycin (see supplemental Fig. 1, available at www.jneurosci.org as supplemental material). As a result, we only observed a weak $\mathrm{spH}$ fluorescence response to stimulation.

In the past, FM dyes have been used specifically to label bulk endocytosis (Richards et al., 2000; Clayton and Cousin, 2008). Here, we did not use FM4-64 to specifically label one type of endocytosis over another; rather we probably labeled all fused membrane with FM4-64 (Fig. 3B). Some FM dyes, such as FM2-10, appear to be excluded from bulk endocytosis pathways (Richards et al., 2000). However, at high enough concentrations $(100 \mu \mathrm{M})$, FM2-10 will also label bulk endocytosis (Clayton and Cousin, 2008). FM4-64 has washout properties similar to FM1-43 (Richards et al., 2000); therefore, FM4-64 should be taken up via all endocytosis pathways.

We explored and compared the nature of the $\mathrm{spH}$ and FM4-64 spots by analyzing several different properties during $100 \mathrm{~Hz}$ stimulation. The only significant difference was the rate of spot dispersal. During recovery after a stimulus train, $\mathrm{spH}$ fluorescence disappeared quickly, indicating both endocytosis and diffusion of $\mathrm{spH}$ out of spots ( $\sim 50 \%$ mobile $\mathrm{spH})$ (Gaffield et al., 2009). FM4-64 spots recovered much more slowly (Fig. 3A), indicating that the majority of the FM4-64 fluorescence increase in these spots was not on the surface. At $100 \mathrm{~Hz}$, due to the strong colocalization of the two signals, we suggest that the majority of endocytosis occurred very near sites of exocytosis. At $40 \mathrm{~Hz}$, however, the majority of FM4-64 was taken up away from $\mathrm{spH}$ spots. At $40 \mathrm{~Hz}$ we could detect the extra exocytosed membrane at spH spots with FM4-64 (a value of $25 \%$ above nonspot areas). We observed an increase in the spH signal of $\sim 50 \%$ in $\mathrm{spH}$ spots compared with nonspot areas. The difference in these two values $(50 \%$ in $\mathrm{spH}$ fluorescence and 25\% in FM4-64 fluorescence) can be attributed to the $\sim 50 \%$ mobile $\mathrm{spH}$ fraction that might have diffused within the membrane and internalized away from the exocytic spH spots (Gaffield et al., 2009).

\section{FM4-64 spots properties are consistent with other measures} of bulk endocytosis

These experiments were performed at physiological temperature $\left(37^{\circ} \mathrm{C}\right)$, using physiological stimulation frequencies for type II muscle fibers (Hennig and Lomo, 1985; Erzen et al., 2000), but for nonphysiological durations (30 s, although FM4-64 spots appeared soon after onset of stimulation). The FM4-64 spots identified here are similar in appearance to FM dye spots found in both fly larvae (Kasprowicz et al., 2008) and fixed snake motor nerve terminals after stimulation (Teng et al., 2007). In both cases, the authors explored the nature of these spots using both fluorescence and electron microscopy, determining that FM dye spots labeled bulk membrane retrieval. The size, locations, and time courses of appearance and decay of the FM4-64 spots are also consistent with previous findings on scattered endocytic hot spots in snake motor nerve terminals (Teng et al., 1999). Moreover, the rapid onset of spot appearance is consistent with the time course of bulk endocytosis in central nerve terminals (Clayton et al., 2008). Finally, the slow dissipation of FM4-64 spots, well after complete internalization of synaptic vesicle membrane (provided by the $\mathrm{spH}$ signal), is also consistent with the slow regeneration and scattering of synaptic vesicles from structures created by bulk endocytosis (Richards et al., 2000, 2003; Lin et al., 2005), although not necessarily measurements of endosome dissipation (Teng et al., 2007). If the FM4-64 spots were not large endosomes, but rather clusters of synaptic vesicles, then the low mobility of these vesicles would likely make them less fusion competent (Gaffield and Betz, 2007). Snake motor nerve terminals equally relied upon bulk endocytosis at frequencies from 0.3 to $30 \mathrm{~Hz}$ (Teng et al., 2007), quite different from our observations in mammalian motor nerve terminals. Possibly, our FM4-64 labeling and spot detection technique preferentially located the largest of cisternae only. Alternatively, endocytosis rates, which are temperature sensitive (Renden and von Gersdorff, 2007; Balaji et al., 2008), might be tuned differently in mammalian and reptilian motor nerve terminals.

We observed prominent endocytosis near the preferred exocytosis sites at $100 \mathrm{~Hz}$ stimulation, suggesting that synaptic vesicle membrane was internalized close to highly used release sites. This type of membrane internalization was much less prevalent at $40 \mathrm{~Hz}$. We were still able to identify spH spots during $40 \mathrm{~Hz}$ stimulation (albeit at a lower intensity) (see also Wyatt and Balice-Gordon, 2008); therefore, tight spatial control over preferred exocytosis and endocytosis locations must not be required for all stimulation levels. The difference in spH spot intensity at $40 \mathrm{~Hz}$ (approximately half that at $100 \mathrm{~Hz}$ ) may indicate the amount of synaptic vesicle membrane that can be efficiently recovered via non-FM4-64 spot pathways. Future measurements, for example, focusing on noncolocalized spots like those that we noted only anecdotally (e.g., points 2, 3 in Fig. 4), may provide a better idea of the relative exocytosis amounts needed to trigger the appearance of a 

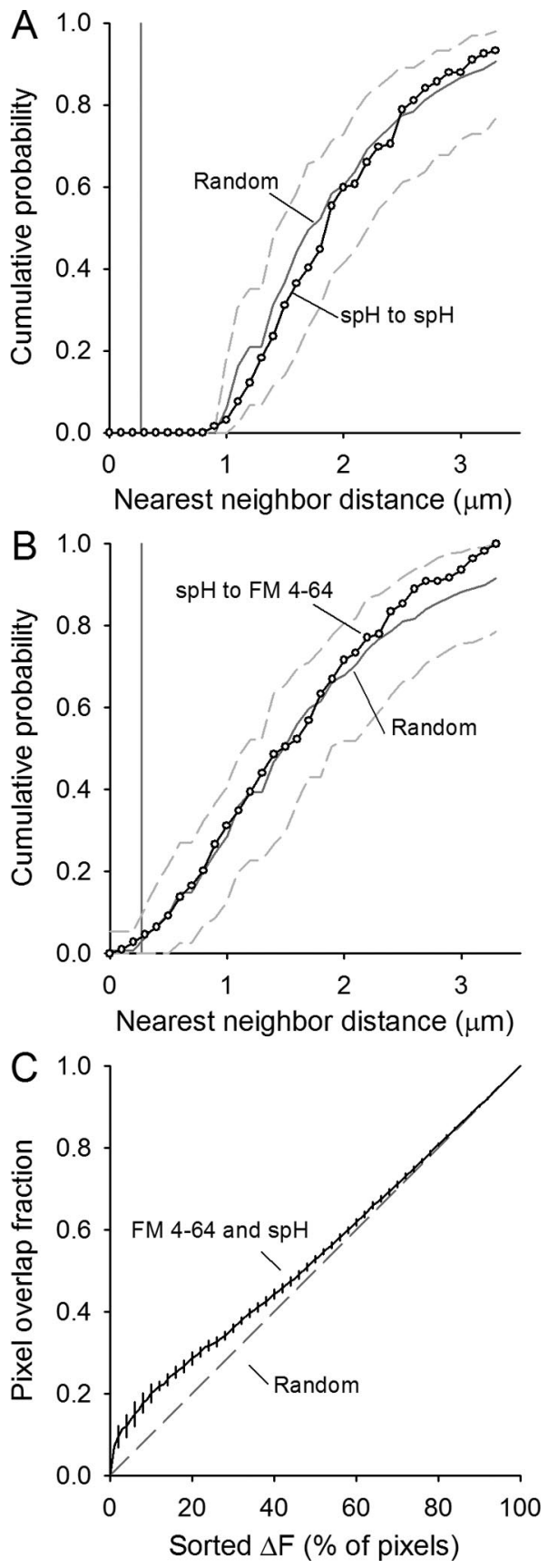

Figure 7. Quantification of spH and FM4-64 spot overlap after moderate stimulation. Plots similar to Figure 5. $A$, Cumulative probability plot of the nearest-neighbor distances for $\mathrm{spH}$ spots identified after a $30 \mathrm{~s}, 40 \mathrm{~Hz}$ stimulation (open circles). Data represent 132 spots from four different terminals. Spots placed randomly in the terminal 1000 times have an average distribution indicated by the dark gray line with $95 \%$ of all random trials falling within the dashed gray lines. Pixel size is indicated by the vertical gray line. $\boldsymbol{B}$, Cumulative probability plot of the nearest-neighbor distances for spH spots to FM4-64 spots (open circles). Spots placed randomly in the terminal 1000 times have an average distribution indicated by the dark gray line with $95 \%$ of all random trials falling within the dashed gray lines. Pixel size is indicated by the vertical gray line. C, The overlap in sorted pixel locations for spH and FM4-64 fluorescence is plotted from $0 \%$ to $100 \%$ of the pixels (black line with error bars). Overlap is only modestly more than expected by random chance (dashed gray line).

nearby endocytic spot. Spatial analysis of other properties related to endocytosis such as intracellular calcium levels (Hosoi et al., 2009), calmodulin activity (Wu et al., 2009), and dynamin I phosphorylation state (Clayton et al., 2009) may help reveal what triggers FM4-64 spot emergence.

\section{A significant number of vesicles cycle through exocytosis/ endocytosis hot spots}

How important are exocytic and endocytic hot spots in the overall function of the motor nerve terminal? The fluorescence in the spots at the end of stimulation was a good indicator of the spot locations, but not necessarily an indicator of the contribution of a spot to overall synaptic vesicle cycling. For example, some spH signal is lost during stimulation due to rapid endocytosis and reacidification (Gaffield et al., 2009). Factoring in this correction, we estimate that $20-30 \%$ of the total $\mathrm{spH}$ signal arose in spots even though the spots comprised only $8-9 \%$ of the terminal area. For the FM4-64 spots, we provided evidence that $20 \%$ of the total FM4-64 fluorescence is located in spot locations at the end of stimulation (Fig. 3A). If we assume that spots disperse at equal rates during and after stimulation, then $20 \%$ is a good approximation for the amount of FM dye taken up in the 7\% of total terminal area occupied by spots. We cannot rule out that more FM4-64 is taken up via the spot pathway, since vesicles budding from putative endosomes might leave the spots during stimulation. Therefore, $20 \%$ is a lower estimate for the amount of membrane uptake via spots at $100 \mathrm{~Hz}$. Consequently, we conclude that both the exocytic pathway visualized by spH spots and the endocytic pathway visualized as FM4-64 spots represent important components of the synaptic vesicle cycle during high-frequency stimulation.

\section{References}

Angaut-Petit D, Molgo J, Connold AL, Faille L (1987) The levator auris longus muscle of the mouse: a convenient preparation for studies of short- and long-term presynaptic effects of drugs or toxins. Neurosci Lett 82:83-88.

Balaji J, Armbruster M, Ryan TA (2008) Calcium control of endocytic capacity at a CNS synapse. J Neurosci 28:6742-6749.

Betz WJ, Bewick GS (1992) Optical analysis of synaptic vesicle recycling at the frog neuromuscular junction. Science 255:200-203.

Betz WJ, Mao F, Bewick GS (1992) Activity-dependent fluorescent staining and destaining of living vertebrate motor nerve terminals. J Neurosci 12:363-375.

Betz WJ, Mao F, Smith CB (1996) Imaging exocytosis and endocytosis. Curr Opin Neurobiol 6:365-371.

Ceccarelli B, Hurlbut WP, Mauro A (1972) Depletion of vesicles from frog neuromuscular junctions by prolonged tetanic stimulation. J Cell Biol 54:30-38.

Ceccarelli B, Hurlbut WP, Mauro A (1973) Turnover of transmitter and synaptic vesicles at the frog neuromuscular junction. J Cell Biol 57:499-524.

Clayton EL, Cousin MA (2008) Differential labelling of bulk endocytosis in nerve terminals by FM dyes. Neurochem Int 53:51-55.

Clayton EL, Evans GJ, Cousin MA (2008) Bulk synaptic vesicle endocytosis is rapidly triggered during strong stimulation. J Neurosci 28:6627-6632.

Clayton EL, Anggono V, Smillie KJ, Chau N, Robinson PJ, Cousin MA (2009) The phospho-dependent dynamin-syndapin interaction triggers activity-dependent bulk endocytosis of synaptic vesicles. J Neurosci 29:7706-7717.

Erzen I, Cvetko E, Obreza S, Angaut-Petit D (2000) Fiber types in the mouse levator auris longus muscle: a convenient preparation to study muscle and nerve plasticity. J Neurosci Res 59:692-697.

Estes PS, Roos J, van der Bliek A, Kelly RB, Krishnan KS, Ramaswami M (1996) Traffic of dynamin within individual Drosophila synaptic boutons relative to compartment-specific markers. J Neurosci 16:5443-5456.

Fernández-Alfonso T, Kwan R, Ryan TA (2006) Synaptic vesicles interchange their membrane proteins with a large surface reservoir during recycling. Neuron 51:179-186.

Gad H, Löw P, Zotova E, Brodin L, Shupliakov O (1998) Dissociation between Ca2+-triggered synaptic vesicle exocytosis and clathrin-mediated endocytosis at a central synapse. Neuron 21:607-616.

Gaffield MA, Betz WJ (2007) Synaptic vesicle mobility in mouse motor nerve terminals with and without synapsin. J Neurosci 27:13691-13700.

Gaffield MA, Tabares L, Betz WJ (2009) The spatial pattern of exocytosis 
and post-exocytic mobility of synaptopHluorin in mouse motor nerve terminals. J Physiol 587:1187-1200.

Hennig R, Lømo T (1985) Firing patterns of motor units in normal rats. Nature 314:164-166.

Heuser JE, Reese TS (1973) Evidence for recycling of synaptic vesicle membrane during transmitter release at the frog neuromuscular junction. J Cell Biol 57:315-344.

Holt M, Cooke A, Wu MM, Lagnado L (2003) Bulk membrane retrieval in the synaptic terminal of retinal bipolar cells. J Neurosci 23:1329-1339.

Hosoi N, Holt M, Sakaba T (2009) Calcium dependence of exo- and endocytotic coupling at a glutamatergic synapse. Neuron 63:216-229.

Kasprowicz J, Kuenen S, Miskiewicz K, Habets RL, Smitz L, Verstreken P (2008) Inactivation of clathrin heavy chain inhibits synaptic recycling but allows bulk membrane uptake. J Cell Biol 182:1007-1016.

Koenig JH, Ikeda K (1989) Disappearance and reformation of synaptic vesicle membrane upon transmitter release observed under reversible blockage of membrane retrieval. J Neurosci 9:3844-3860.

Lin MY, Teng H, Wilkinson RS (2005) Vesicles in snake motor terminals comprise one functional pool and utilize a single recycling strategy at all stimulus frequencies. J Physiol 568:413-421.

Martin AR (1955) A further study of the statistical composition on the endplate potential. J Physiol 130:114-122.

Miesenböck G, De Angelis DA, Rothman JE (1998) Visualizing secretion and synaptic transmission with $\mathrm{pH}$-sensitive green fluorescent proteins. Nature 394:192-195.

Miller TM, Heuser JE (1984) Endocytosis of synaptic vesicle membrane at the frog neuromuscular junction. J Cell Biol 98:685-698.

Ng M, Roorda RD, Lima SQ, Zemelman BV, Morcillo P, Miesenböck G (2002) Transmission of olfactory information between three populations of neurons in the antennal lobe of the fly. Neuron 36:463-474.

Renden R, von Gersdorff H (2007) Synaptic vesicle endocytosis at a CNS nerve terminal: faster kinetics at physiological temperatures and increased endocytotic capacity during maturation. J Neurophysiol 98:3349-3359.

Richards DA, Guatimosim C, Betz WJ (2000) Two endocytic recycling routes selectively fill two vesicle pools in frog motor nerve terminals. Neuron 27:551-559.

Richards DA, Guatimosim C, Rizzoli SO, Betz WJ (2003) Synaptic vesicle pools at the frog neuromuscular junction. Neuron 39:529-541.
Roos J, Kelly RB (1999) The endocytic machinery in nerve terminals surrounds sites of exocytosis. Curr Biol 9:1411-1414.

Sankaranarayanan S, Ryan TA (2001) Calcium accelerates endocytosis of vSNAREs at hippocampal synapses. Nat Neurosci 4:129-136.

Smith CB, Betz WJ (1996) Simultaneous independent measurement of endocytosis and exocytosis. Nature 380:531-534.

Smith SM, Renden R, von Gersdorff H (2008) Synaptic vesicle endocytosis: fast and slow modes of membrane retrieval. Trends Neurosci 31:559-568.

Tabares L, Ruiz R, Linares-Clemente P, Gaffield MA, Alvarez de Toledo G, Fernandez-Chacón R, Betz WJ (2007) Monitoring synaptic function at the neuromuscular junction of a mouse expressing synaptopHluorin. J Neurosci 27:5422-5430.

Takei K, Mundigl O, Daniell L, De Camilli P (1996) The synaptic vesicle cycle: a single vesicle budding step involving clathrin and dynamin. J Cell Biol 133:1237-1250.

Teng H, Wilkinson RS (2000) Clathrin-mediated endocytosis near active zones in snake motor boutons. J Neurosci 20:7986-7993.

Teng H, Cole JC, Roberts RL, Wilkinson RS (1999) Endocytic active zones: hot spots for endocytosis in vertebrate neuromuscular terminals. J Neurosci 19:4855-4866.

Teng H, Lin MY, Wilkinson RS (2007) Macroendocytosis and endosome processing in snake motor boutons. J Physiol 582:243-262.

Wienisch M, Klingauf J (2006) Vesicular proteins exocytosed and subsequently retrieved by compensatory endocytosis are nonidentical. Nat Neurosci 9:1019-1027.

Wu LG, Ryan TA, Lagnado L (2007) Modes of vesicle retrieval at ribbon synapses, calyx-type synapses, and small central synapses. J Neurosci 27:11793-11802.

Wu XS, McNeil BD, Xu J, Fan J, Xue L, Melicoff E, Adachi R, Bai L, Wu LG (2009) $\mathrm{Ca}(2+)$ and calmodulin initiate all forms of endocytosis during depolarization at a nerve terminal. Nat Neurosci 12:1003-1010.

Wyatt RM, Balice-Gordon RJ (2008) Heterogeneity in synaptic vesicle release at neuromuscular synapses of mice expressing synaptopHluorin. J Neurosci 28:325-335.

Xu YF, Autio D, Rheuben MB, Atchison WD (2002) Impairment of synaptic vesicle exocytosis and recycling during neuromuscular weakness produced in mice by 2,4-dithiobiuret. J Neurophysiol 88:3243-3258.

Zhang Q, Li Y, Tsien RW (2009) The dynamic control of kiss-and-run and vesicular reuse probed with single nanoparticles. Science 323:1448-1453. 